\title{
Dual HF radar study of the subauroral polarization stream
}

\author{
R. A. Makarevich and P. L. Dyson \\ Department of Physics, La Trobe University, Victoria, 3086, Australia \\ Received: 2 August 2007 - Revised: 6 December 2007 - Accepted: 12 December 2007 - Published: 2 January 2008
}

\begin{abstract}
The dual HF radars comprising the Tasman International Geophysical Environment Radar (TIGER) system often observe localized high-velocity F-region plasma flows $(\geq 1500 \mathrm{~m} / \mathrm{s})$ in the midnight sector (20:00-02:00 MLT) at magnetic latitudes as low as $\Lambda=60^{\circ} \mathrm{S}$. The flow channels exhibit large variability in the latitudinal extent and electric field strength, and are similar to the subauroral polarization stream or SAPS, a plasma convection feature thought to be related to the polarization electric field due to the charge separation during substorm and storm development. In this study, the 2-D plasma drift velocity within the channel is derived for each of the two TIGER radars from the maximum velocities measured in all 16 radar beams within the latitudinally narrow channel, and the time variation of the subauroral electric field is examined near substorm onset. It is demonstrated that the flow channel often does not have a clear onset, rather it manifests differently in different phases of its evolution and can persist for at least two substorm cycles. During the growth phase the electric fields within the flow channel are difficult to distinguish from those of the background auroral convection but they start to increase near substorm onset and peak during the recovery phase, in contrast to what has been reported previously for auroral convection which peaks just before the substorm onset and falls sharply at the substorm onset. The response times to substorm onset range from -5 to +40 min and show some dependence on the substorm location with longer delays observed for substorms eastward of the radars' viewing area. The propagation velocity of the high-velocity region is also investigated by comparing the observations from the two closely-spaced TIGER radars. The observations are consistent with the notion that the polarization electric field is established with the energetic ions drifting westward and equatorward from the initial substorm injection. The ion injection front can precede that of the electrons and hence substorm onset resulting in a negative response time of a few minutes.
\end{abstract}

Keywords. Ionosphere (Electric fields and currents; Plasma convection) - Magnetospheric physics (Storms and substorms)

Correspondence to: R. A. Makarevich

(r.makarevich@latrobe.edu.au)

\section{Introduction}

In-situ plasma convection observations by low-orbit satellites identified latitudinally narrow regions of very strong westward plasma flows termed Polarization Jets or PJ (Galperin et al., 1973), Subauroral Ion Drifts or SAID (Spiro et al., 1979), and Subauroral Electric Fields or SAEF (Karlsson et al., 1998). PJ/SAID/SAEF were proposed to occur whenever the inner plasma sheet electrons and ions separate radially establishing the poleward polarization electric field in the low conductivity region equatorward of the electron precipitation (Southwood and Wolf, 1978). This separation frequently occurs soon after the substorm injection with the plasmasheet ions penetrating deeper into the inner magnetosphere on the dusk side so that PJ/SAID are often said to be a substorm-related phenomenon (e.g. Anderson et al., 1993). A similar situation occurs during magnetospheric storms when strong convection electric field produces a substantial radial separation between the inner edges of the plasmasheet ions and electrons. Consequently, wider regions of enhanced plasma convection covering up to $5^{\circ}$ magnetic latitude were shown to be regularly observed by the Millstone Hill incoherent scatter radar (MH ISR) during disturbed conditions equatorward of the electron precipitation and auroral convection cells (Foster and Vo, 2002) and an encompassing term Subauroral Polarization Stream or SAPS has been proposed (Foster and Burke, 2002).

Similar substorm-related features in plasma convection have been identified using coherent VHF radars at subauroral latitudes (Unwin and Cummack, 1980; Freeman et al., 1992) and most equatorward radars in the Super Dual Auroral Radar Network (SuperDARN or SD) of coherent HF radars (Parkinson et al., 2003; Koustov et al., 2006; Oksavik et al., 2006). Parkinson et al. (2003) have observed substorm-associated enhancements in plasma convection with the Bruny Island SD radar and introduced a new term Auroral Westward Flow Channel or AWFC, while Koustov et al. (2006) have stopped short of directly associating AWFC observed with the King Salmon SD radar and SAPS. Finally, Oksavik et al. (2006) have used satellite observations of auroral activity and in situ electric fields to conclude that flow channels seen by the mid-latitude Wallops Island SD radar were manifestations of SAPS.

Published by Copernicus Publications on behalf of the European Geosciences Union. 
A range of observational techniques and different criteria for identification of features in the data used by researchers have made the task of studying the relationship between SAPS and substorms somewhat difficult and hence mechanisms responsible for the SAPS generation. In this regard, an important question is the SAPS onset timing in relation to the substorm onset. Anderson et al. (1993) have demonstrated that SAID occur more than $30 \mathrm{~min}$ after the substorm onset and almost always during the substorm recovery phase. In the same study, however, in several cases enhanced ion drifts below the threshold value of $1000 \mathrm{~m} / \mathrm{s}$ (used for SAID identification) were observed during expansion phase. Delays between substorm onset and SAID as low as $10 \mathrm{~min}$ were observed by Unwin and Cummack (1980) and Karlsson et al. (1998) with the empirical model of Galperin (2002) implying the generation of PJ within 5-10 min from substorm onset. Furthermore, Parkinson et al. (2003) showed that an AWFC appeared simultaneously with (within 2 min of) substorm onset as determined from magnetometer records. On the other hand, Koustov et al. (2006) considered observations of SAPS-like plasma flows by the King Salmon SD radar and concluded that substantial flows were generated some tens of min after substorm onset as determined from optical, magnetometer Pi2 pulsation and energetic particle observations.

It is clear from this quick literature survey that the relationship between SAPS and substorms requires further investigation. Perhaps one important clue is provided by the recent studies that demonstrated a significant variability in the plasma flow velocity within SAPS as well as in the latitudinal extent of SAPS (Foster et al., 2004; Oksavik et al., 2006; Koustov et al., 2006). Coherent backscatter observations from SD radars such as Bruny Island, King Salmon or Wallops Island are advantageous since measurements are provided on quasi-continuous basis over extended areas of the ionosphere with relatively good spatial $(15-45 \mathrm{~km})$ and temporal resolutions (1-2 min). The aim of this study is to investigate the temporal evolution of the SAPS plasma convection velocity/electric field around substorm onset. The specific objectives are (1) to determine the typical time variation of the plasma convection velocity; (2) to find whether different features observed in the data can be manifestations of the same phenomenon at different phases of its temporal evolution; and (3) to study time delays between substorm onset and SAPS onset.

\section{Observations}

\subsection{Dual TIGER radars}

In this study, data from the two coherent HF radars that constitute the Tasman International Geophysical Environment Radar (TIGER) dual system are considered. One radar is located on Bruny Island (BI), Tasmania (geographic $43.4^{\circ} \mathrm{S}$, $147.2^{\circ} \mathrm{E}$, with the field-of-view (FoV) centered at boresite of $180^{\circ} \mathrm{E}$ of $\mathrm{N}$ ) and the other radar is near Invercargill, New Zealand (NZ) $\left(46.5^{\circ} \mathrm{S}, 168.4^{\circ} \mathrm{E}\right.$, with boresite of $\left.227.9^{\circ}\right)$. The TIGER BI radar has been operational since 1999 and the TIGER NZ radar, also known as Unwin, has started operations in November 2004. The two radars constitute the most equatorward pair with overlapping FoVs in the SuperDARN array with F-region echoes at magnetic latitudes (MLAT) as low as $\Lambda=60^{\circ} \mathrm{S}$ often observed by both radars.

The TIGER radars are similar to other SuperDARN radars, technically and operationally, as described by Greenwald et al. (1995) and more recently by Chisham et al. (2007). Each radar scans through 16 azimuthal directions (radar beams 0 15 ) in $3.25^{\circ}$ steps, covering an azimuth range of $\sim 52^{\circ}$. The radars measure a 17-lag ACF from which estimates of the Doppler velocity, power, and spectral width of ionospheric echoes in 75 range bins for each of the 16 radar beams are obtained. The Doppler velocity measured corresponds to the line-of-sight or l-o-s irregularity velocity and is, by convention, positive for irregularities propagating towards the radar. The range gate length was $45 \mathrm{~km}$, with the first range gate at $180 \mathrm{~km}$. The sounding frequency is usually fixed somewhere between 9 and $18 \mathrm{MHz}$.

\subsection{Event selection and data post-processing}

To investigate the plasma flow velocity variation around substorm onset the 6 events were selected using the following algorithm. All ground-scatter echoes were excluded first using the standard SD criteria of low Doppler velocity and low spectral width. The ionospheric echo dataset was cleaned excluding echoes with low signal-to-noise ratio (SNR) $P<3 \mathrm{~dB}$, large spectral width $W>500 \mathrm{~m} / \mathrm{s}$, and very large speed $V>2000 \mathrm{~m} / \mathrm{s}$. The E-region echoes at short ranges $r<765 \mathrm{~km}$ and F-region echoes at very large ranges $r \geq 1980 \mathrm{~km}$ were also excluded. The database of substorm onsets (Frey and Mende, 2006) was employed for initial event search and the period of November 2004 December 2005 was considered to find events with simultaneous data from both TIGER radars (November 2004 present) and substorm onset information (January 2003 - December 2005 in Southern Hemisphere). Isolated substorms (>90 min difference) should have been observed between 08:00-12:00 UT ( 20:00-24:00 MLT) near the TIGER FoV $\left(42.5-62.5^{\circ} \mathrm{S}, 110-200^{\circ} \mathrm{E}\right)$. The other criterion used was that the zonally-looking TIGER NZ radar should observe more than 1500 echoes with Doppler velocities below $-500 \mathrm{~m} / \mathrm{s}$ (westward velocity exceeding $500 \mathrm{~m} / \mathrm{s}$ ) between 08:00-14:00 UT ( 20:00-02:00 MLT) and $\Lambda=59^{\circ}-66^{\circ} \mathrm{S}$. This criterion was adopted to find events with high SAPSlike plasma flow velocities and is similar to other criteria for velocity used in the past (e.g. Spiro et al., 1979; Karlsson et al., 1998; Koustov et al., 2006). To study time variation near substorm onset another restriction was introduced, i.e. that both TIGER radars should observe no less than 400 echoes in both $10 \mathrm{~min}$ preceding substorm onset and $10 \mathrm{~min}$ following 
the onset. The resulting events were examined with respect to location of the region with enhanced velocities relative to the Defense Meteorology Satellite Program (DMSP) precipitation boundaries as illustrated below similar to other recent studies (Koustov et al., 2006; Oksavik et al., 2006).

Table 1 shows the list of events with substorm onset time and location and $K_{p}$ indices indicated. The second column shows the scanning mode of both TIGER radars. In the common mode, the radars scan through successive beam positions (from 0 to 15) with integration time at each position of $7 \mathrm{~s}$ with the total scan time of $2 \mathrm{~min}$. In the fast mode, the integration and resolution are 3 and $60 \mathrm{~s}$, respectively. During 4 events, TIGER operated in a special "Trident" mode. This is a "multiple-camping-beam" mode with integration time of $3 \mathrm{~s}$ and total scan time of $\sim 100 \mathrm{~s}$. The 3 camping beams were sampled 6 times in a cycle, with all other beams sampled once.

In the selection process described above, the 5 numbers (number of echoes $10 \mathrm{~min}$ before and after substorm onset for both TIGER radars and number of high-velocity echoes for TIGER NZ) had to be above their respective threshold values for each event. To ensure that our selection algorithm was not biased towards events with higher than normal beam sampling rate, for each event all 5 numbers were "normalised" by counting echoes in camping beams, dividing by 6 and adding echoes in non-camping beams. After the "renormalization", only one number for one event has dropped significantly below the threshold value. This calculation and further examination of individual events clearly show that the selection procedure employed has produced true SAPS-like events rather than events with high echo counts simply due to high rate of camping beam sampling.

\subsection{TIGER observations of SAPS}

Figure 1 shows the TIGER FoV with Tasmania in the topmiddle part and South Island of New Zealand in the top-right corner of each panel. The green curves are the Polar AngloAmerican Conjugate Experiment (PACE) lines of equal magnetic latitude $\Lambda=60^{\circ} \mathrm{S}$ and $70^{\circ} \mathrm{S}$. TIGER BI (NZ) observes in directions that are more perpendicular (parallel) to magnetic $L$-shells with beam 0 being the westernmost beam (left in Fig. 1).

The TIGER 5-min velocity maps for 8 February 2005 for selected frames are shown in Fig. 1. For each frame, the average velocity was calculated for cells with 2 or more echoes. The time of the frame in UT and in minutes from substorm onset is indicated in the left-top corner of each panel. The DMSP pass with the cross-track ion drift velocities given as coloured vectors is shown in panel (b). The DMSP trajectory was projected from a satellite altitude of around $860 \mathrm{~km}$ down to $200 \mathrm{~km}$ along the IGRF magnetic field line to account for different altitudes of TIGER and DMSP measurements. The velocity colour scale is the same for both TIGER and DMSP and is given in panel (a).
Table 1. Selected periods of the TIGER observations. The columns 3-6 show the time in UT, geographic latitude (in degrees South), geographic longitude (in degrees East) and PACE magnetic latitude (in degrees South) of the substorm onset. The column 7 shows the $K_{p}$ indices between 06:00 UT and 15:00 UT.

\begin{tabular}{|c|c|c|c|c|c|c|}
\hline \multirow{2}{*}{ Date } & \multirow[t]{2}{*}{ Mode } & \multicolumn{4}{|c|}{ Substorm Onset } & \multirow{2}{*}{$K_{p}$} \\
\hline & & Time & GGlat & GGlon & MLAT & \\
\hline 20041121 & fast & 09:02 & 57.6 & 155.8 & 68.1 & $34-4-$ \\
\hline 2004 & trid & 10:06 & 55.5 & 131.0 & 69. & \\
\hline 2005 & $\operatorname{tri}$ & 08:28 & 59.9 & 180.0 & 64.7 & $4-3+4-$ \\
\hline 20050209 & tric & 09:17 & 56.7 & 174.4 & 63.0 & $5-44-$ \\
\hline 20050211 & trid & $10: 45$ & 55.9 & 156.8 & 66.2 & $2+3+2+$ \\
\hline 20050219 & com & $11: 35$ & 54.7 & 131.4 & 68.8 & $3+44-$ \\
\hline
\end{tabular}

The convection was moderate before the substorm onset, panel (a), with maximum velocities of $\sim 200 \mathrm{~m} / \mathrm{s}$. In panel (b), 17 min after substorm onset, the TIGER NZ velocity at $\sim 61^{\circ} \mathrm{S}$ increased to $500 \mathrm{~m} / \mathrm{s}$ with the DMSP velocities of $500-700 \mathrm{~m} / \mathrm{s}$ (one has to bear in mind slightly different locations and look directions for TIGER and DMSP). Importantly, the location of electron precipitation boundary (yellow circle) determined from in-situ particle flux data from SSJ/4 particle detector indicates that an enhanced plasma convection channel evident in both TIGER NZ and DMSP data was located equatorward of the electron precipitation region. In panels (c) and (d), 37 and 67 min after substorm onset, the region with enhanced convection widens and enhanced velocities become evident in the TIGER BI observations in panel (d). It is important to note that the TIGER NZ velocity showed some increase soon after the substorm whereas TIGER BI showed a similar increase only after $40 \mathrm{~min}$. This feature is examined in more detail later in Sect. 2.4.

Figure 2 presents the TIGER velocity observations on 21 November 2004 in the same format as Fig. 1. The substorm onset as determined by IMAGE occurred at 09:02 UT at a location shown by the black-yellow square. Before substorm onset, panel (a), velocities were around $100 \mathrm{~m} / \mathrm{s}$ in both radars. The DMSP ion drift data showed some enhancement in westward velocities with the largest velocities observed equatorward of the electron precipitation boundary. In panel (b), 13 min after substorm onset, equatorward of the substorm onset location the BI velocity is $400-500 \mathrm{~m} / \mathrm{s}$ with TIGER NZ observing lower velocities of $200 \mathrm{~m} / \mathrm{s}$. No enhanced velocity was observed on the edges of the combined FoV (beams $0-3$ of TIGER BI and beams $12-15$ of TIGER NZ). In panel (c), 43 min after substorm onset, the velocity map shows a clear signature of the latitudinally narrow $\left(\sim 2^{\circ}\right)$ channel of enhanced convection extending in longitude across the entire TIGER FoV. Finally, in panel (d), 93 min after substorm onset, the flow channel is much narrower with very sharp edges, in particular in the TIGER NZ observations. The DMSP F15 pass to the east of TIGER 

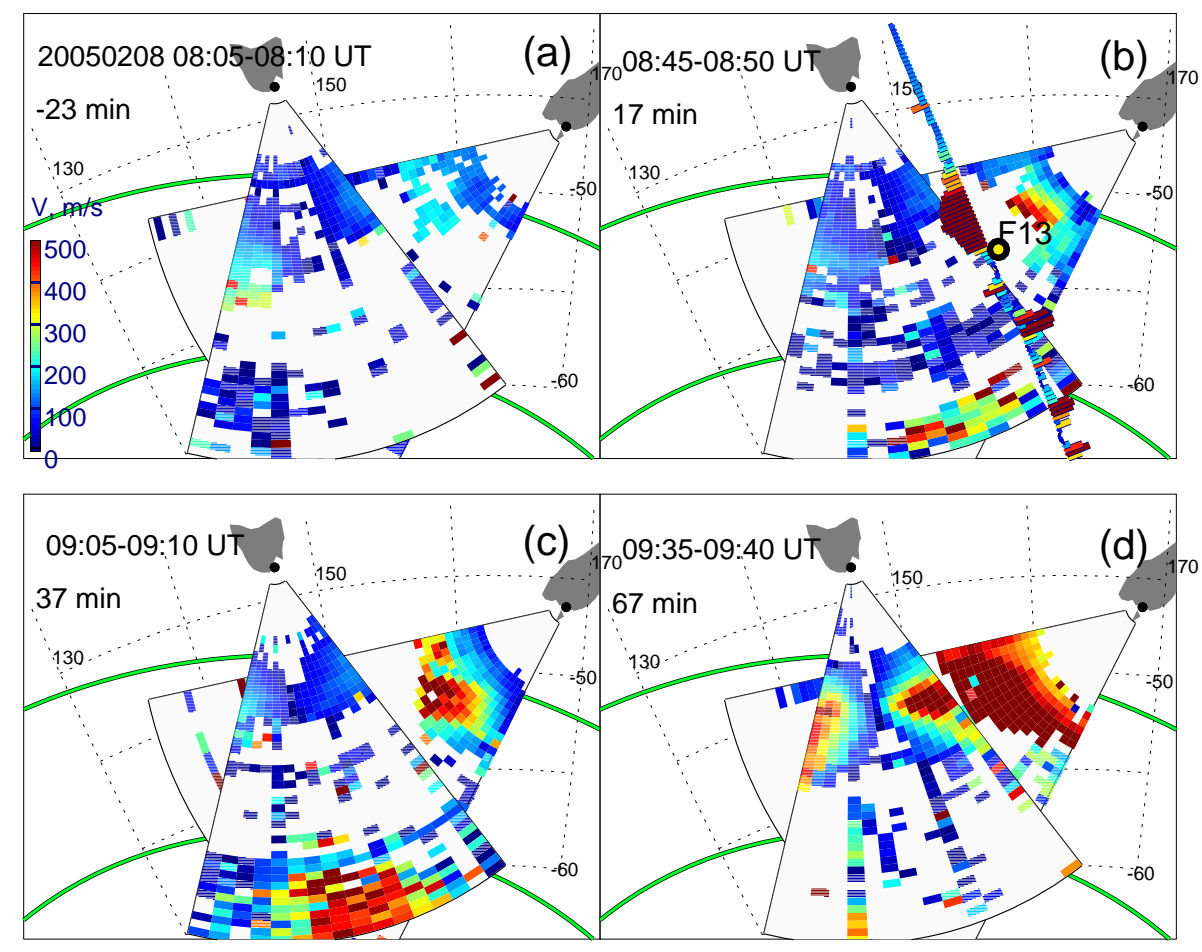

Fig. 1. TIGER 5-min velocity maps for 8 February 2005. Positive line-of-sight velocities are indicated by cells filled with solid colour, while negative velocities are shown by cells filled with horizontal lines. The TIGER NZ velocity is reversed. Green lines are the PACE lines of equal magnetic latitudes $\left(\Lambda=60^{\circ} \mathrm{S}\right.$ and $\left.70^{\circ} \mathrm{S}\right)$. The 5-min time interval and the difference between the substorm onset and the start time of the frame are shown in the top-left corner. The DMSP F13 pass with the simultaneously measured perpendicular ion velocities are indicated by colour vectors. The colour bar for the TIGER and DMSP velocities is shown in panel (a). The yellow dot with a black circle around is the electron precipitation boundary called ble. For both TIGER BI and NZ, beam 0 (15) is the leftmost (rightmost) beam of the radar.

NZ showed a classical PJ/SAID signature with the ion crosstrack velocities well above $1000 \mathrm{~m} / \mathrm{s}$. Maximum DMSP velocities were, again, observed equatorward of the electron precipitation region. A similar feature was observed by DMSP F13 west of TIGER.

\subsection{Flow velocity variation with time}

Examination of the velocity maps in Figs. 1 and 2 shows that the latitudinal position of the flow channel, its width and the flow speed within the channel exhibit significant variations with time. To investigate in more detail the flow speed/electric field strength temporal evolution the following semi-automatic method of velocity determination was developed. For each 5-min frame and for each radar beam, all local maxima in the velocity versus range were found in the magnetic latitude range $\Lambda=59^{\circ}-66^{\circ} \mathrm{S}$. The maxima farther from the previous position of the flow channel than $2^{\circ}$ for BI and $1^{\circ}$ for $\mathrm{NZ}$ were excluded. The main velocity maximum within the specified range was taken as the maximum 1-o-s velocity along the radar beam. These maxima were shown by the tiny green circles in Fig. 2. The larger latitude range of $2^{\circ}$ for TIGER BI was selected because the tracing algorithm was often loosing track of the channel in the BI data if the same range of $1^{\circ}$ in MLAT was used for both radars. The main reason for this is that TIGER BI observes in more meridional directions, which results in smaller 1-o-s velocities measured for mostly zonal plasma flows. As the velocity maxima along each beam were determined, smaller velocity values for TIGER BI resulted in larger random variations in the latitudinal position of the maxima between successive frames. To keep track of the channel position for both radars, a $2^{\circ}$-wide range in MLAT was used for TIGER BI.

Figure 3 shows individual Doppler velocities measured by the TIGER (a) BI and (b) NZ radars versus the $L$-shell angle for the same frame as in Fig. 2c. All azimuths were measured clockwise from the geographic north and the $L$-shell angle was defined as a difference between the azimuth of observations and the azimuth of eastern sector of the PACE magnetic parallel at $200 \mathrm{~km}$. Thus $L$-shell angle is always positive and for TIGER BI it takes the range of values from $\phi \cong 45^{\circ}$ (beam 15) to $105^{\circ}$ (beam 0 ). The range for TIGER $\mathrm{NZ}$ is from $\phi \cong 100^{\circ}$ (beam 15) to $160^{\circ}$ (beam 0). The dots in Fig. 3 are colour-coded in magnetic latitude according to the colour scheme shown in panel (a). The data from all 16 beams are collected here and the points exhibit a clear clustering according to the beam number as illustrated by the 

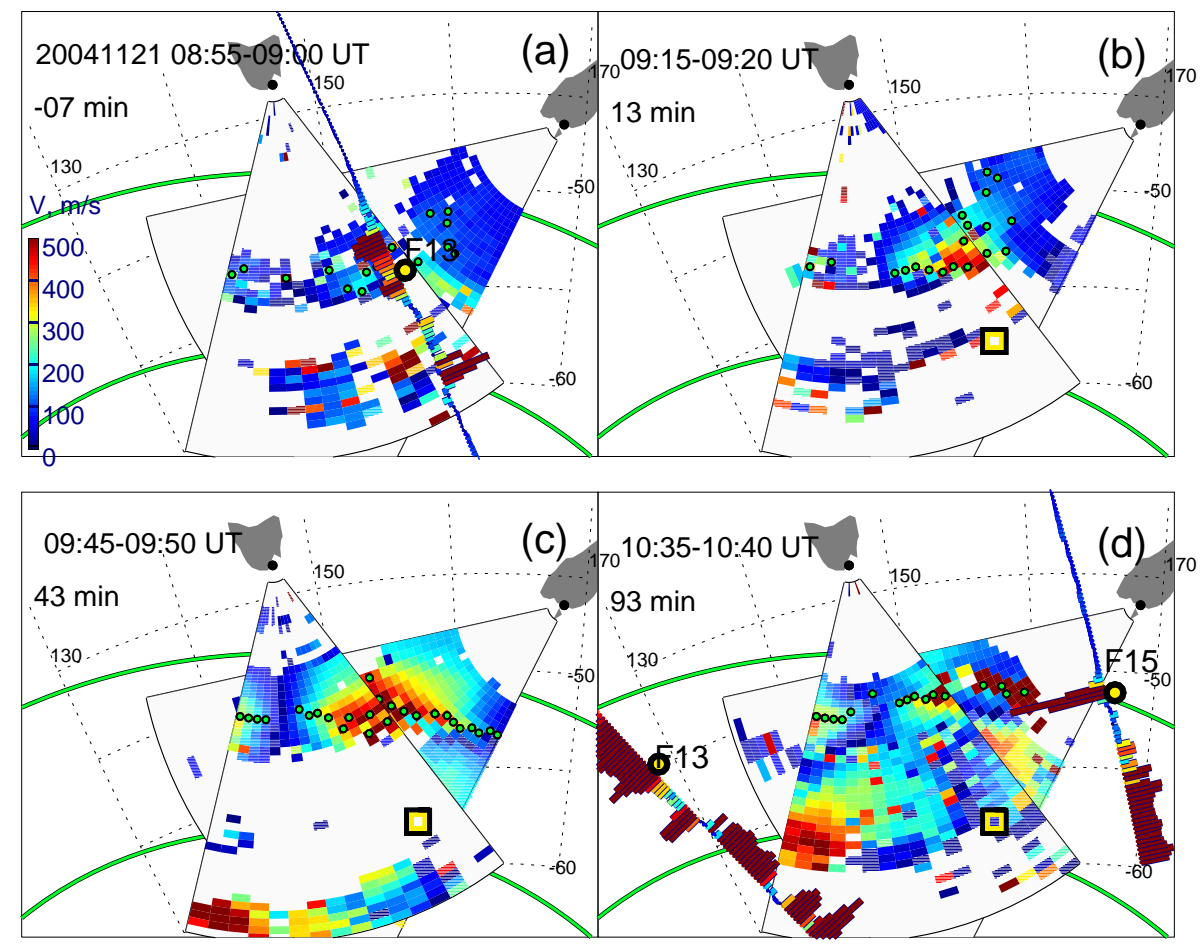

Fig. 2. The same as Fig. 1 but for 21 November 2004. For each radar beam, the radar range with maximum velocity is shown by a tiny green circle. The black-yellow square in panels (b)-(d) shows the location of the substorm onset.

black lines in both panels that show the average velocity in a single beam for each radar. The diamonds show the maximum velocities for up to 16 beams determined as described above. They are also colour-coded in magnetic latitude $\Lambda$.

The diamonds are generally at the top or bottom (depending on the velocity sign) of the cluster of points corresponding to the data from a specific beam. For this frame, the diamonds also generally follow the trend expected for an $L$ shell-aligned westward plasma flow with reversal in the BI velocities occurring at $\phi \cong 90^{\circ}$ and negative NZ velocities (receding irregularities). This trend is approximated by the cosine function $V_{0} \cos \left(\phi+\phi_{0}\right)$ with coefficients $V_{0}$ and $\phi_{0}$ representing the plasma flow speed and direction within the flow channel, respectively. For each 5-min frame, the coefficients $V_{0}$ and $\phi_{0}$ were found from the non-linear least squares best fit to the maximum velocities with initial values for $V_{0}$ and $\phi_{0}$ taken from the previous frame. The average latitudinal position in $\Lambda$ of the flow channel (over all beams) is also given in each panel. This "all-beam cosine approximation" implies that the flow is mostly homogeneous in longitude/MLT within the channel in the FoV for each TIGER radar. Similar methods were employed by Ruohoniemi et al. (1989) and more recently by Makarevitch et al. (2004).

The "MLT-homogeneity" assumption sometimes breaks as for the 4 westernmost (low-number) beams of TIGER NZ on the right of panel (b). For some beams, the algorithm has selected the velocity maximum which showed a significant jump in magnetic latitude as compared to neighboring beams which was nevertheless within $1^{\circ}$ or $2^{\circ}$ of the previous position and so met the criteria in magnetic latitude. In all such cases, the "outlying" maxima were manually excluded from the best cosine fit shown by the solid line in Fig. 3. All maximum velocities that were $<100 \mathrm{~m} / \mathrm{s}$ in magnitude were also excluded. In the following, only the frames that had more than 7(5) valid maxima for BI (NZ) were considered. The condition for NZ was less restrictive given that it was generally more difficult to satisfy the "MLT-homogeneity" condition for the zonally-looking NZ radar, as in Fig. 3.

Figure 4 shows the results of velocity fitting for the entire event of 21 November 2004. The vertical bars are the uncertainties in the fitted parameters $V_{0}$ and $\phi_{0}$ that take into account standard deviations in the average velocities shown by diamonds in Fig. 3 (these uncertainties were not shown in Fig. 3 to make the diagram more readable). One can notice that the plasma flow speed in panel (a) exhibited an initial increase for both radars some time after the substorm onset shown by the vertical line (both green and blue lines start to go up). No delay was observed in the BI data (green line goes up immediately after the substorm onset), while the NZ velocity shows a delay of about $15-20 \mathrm{~min}$, in agreement with Fig. 2. After that the speed variations were quite similar for both radars. The velocity reached maximum at 10:30-11:00 UT or 90-120 min after substorm onset, again in agreement with the individual velocity maps (Fig. 2d). The flow direction was on average roughly along the $L$-shell, although uncertainties in the NZ data were quite large after 


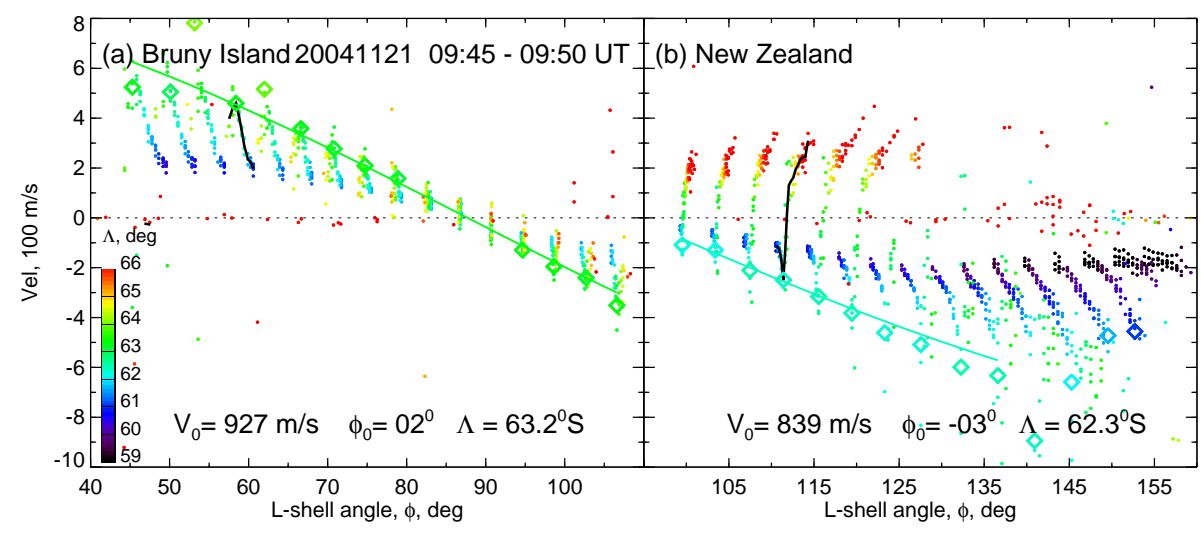

Fig. 3. Scatter plot of the Doppler velocity versus $L$-shell angle $\phi$ for 21 November 2004, 09:45-09:50 UT for the TIGER (a) Bruny Island and (b) New Zealand radars. The two black lines show velocity variation in beam 12 for BI and NZ radars. The diamonds are maxima of the averaged (over the frame time interval) velocity in all radar beams as described in the text. The points and diamonds are colour-coded in magnetic latitude $\Lambda$ as indicated in panel (a). The solid line represents the best fit, $V_{0} \cos \left(\phi+\phi_{0}\right)$, to diamonds. The fitting coefficients $V_{0}$ and $\phi_{0}$ and the magnetic latitude of cells with velocity maxima averaged over all beams are given in each panel.

10:00 UT, panel (b). The flow channel exhibited overall equatorward motion before about 11:00 UT. The latitudinal positions observed by the two radars differed by $\sim 1^{\circ}$ with TIGER BI showing a slightly poleward location, panel (c). One can also observe some rotation of the channel in the BI data with the easternmost beams 10-15 (green dash-dotted line) detecting a more equatorward location. This is in agreement with the TIGER NZ observations: TIGER NZ is located eastward of TIGER BI and so some difference between latitudinal positions can be explained by a finite rotation of the flow channel from the $L$-shell direction (the flow channel is located at lower latitudes at later MLTs).

The fitted "all-beam" velocities have been calculated for all 6 events considered and in Fig. 5 the results for the fitted plasma flow speed $V_{0}$ are presented as a function of time from the substorm onset. The TIGER BI (NZ) fitted velocity is shown by the red (black) line. To put the results of the fitting into the context of actual l-o-s velocity measurements and check whether they make sense, Fig. 5 also shows all TIGER NZ velocity 1-o-s measurements colour-coded in magnetic latitude as shown in panel (b). The events are sorted according to the shape of the velocity time variation rather than sequentially as in Table 1 . The substorm onsets are shown by the vertical line. The digits on the left show the geographic longitude of the substorm onset. One should bear in mind that the fitted velocity is calculated using observations within the channel only, while Fig. 5 shows all velocity measurements.

In all events, the fitted NZ velocity (black) generally follows the bottom envelope of the NZ 1-o-s velocity. Occasionally, this feature is not observed as in panel (a), $40 \mathrm{~min}$ before substorm, when a relatively short-lived velocity spike is seen. In all such cases, the diagrams similar to Fig. 3 were checked to confirm that the calculated fitted velocity was of reasonable quality. In all events but the first one, the veloc- ity exhibited a marked increase near substorm onset. On 25 November 2004, panel (a), velocity started to increase well before the substorm (40-60 $\mathrm{min}$ ) so that near substorm onset velocities were already elevated to $\sim 1000-1400 \mathrm{~m} / \mathrm{s}$. After substorm onset no significant increase in velocity was observed, rather some decrease was seen so that $20 \mathrm{~min}$ after substorm onset velocities were $600-800 \mathrm{~m} / \mathrm{s}$. One should note though that this event was characterized by a particular low magnetic latitude of the flow channel (as low as $60^{\circ}$ ). It is possible that some of the decrease seen was simply due to the fact that the flow channel moved to closer ranges corresponding to E-region scatter since the E-region irregularity velocity is known to be significantly reduced compared to F-region convection velocities (Makarevitch et al., 2004).

In the events that did show an increase near substorm onset, the trends differed from event to event, with two events, panels (b) and (c), showing velocity reaching maximum within 20-40 min from substorm and with three other events, panels (d)-(f), showing more gradual increase with some variation. On 19 February 2005, panel (b), velocities started to increase $\sim 5 \mathrm{~min}$ before substorm onset. On the other hand, positive delays between substorm onset and the timing of the initial increase in the TIGER NZ velocity were observed in all other events but one. One should note though that during the latter event, on 09 February 2005, panel (e), the velocity increase less than $10 \mathrm{~min}$ after substorm onset was small and well within uncertainties. A large positive delay of $>40$ min was observed in the BI velocities on 8 February 2005, panel (f).

The last observation is consistent with the presentation of Fig. 1c that showed that 37-42 min after substorm onset the BI velocities were comparable with pre-substorm levels and smaller than velocities measured by TIGER NZ. The opposite delay was observed in Fig. 2, with the TIGER NZ velocity being smaller than that measured by TIGER BI 13 
min into the substorm expansion phase, panel (b), despite the more zonal look direction of TIGER NZ. The BI velocity increase preceding that in the NZ velocity was also observed in Fig. 5d. Moreover, from Fig. 5 it appears that a similar feature is present in panel (b)-(d), with the red line to the left of the black line near substorm onset. The opposite effect is seen in panels (e)-(f). Looking at the geographic longitude of the substorm onset (left of vertical line), one can notice that the last two substorms were detected a considerable distance east of the TIGER FoV, whereas in panels (b)-(d) the substorm locations were at or west of that shown in Fig. 2b, and hence either within or westward of the FoV.

To further test that the observed delays between fitted velocities inferred from the two TIGER radars represent the data reasonably well, the BI and NZ velocities were compared directly. Taking into account that individual velocities in Fig. 5 are the 1-o-s (cosine) components of the plasma velocity and that the flow within the channel is to the first approximation along the $L$ shell, the TIGER velocities measured were divided by the cosine component of the $L$-shell angle $\phi$ to estimate 2-D plasma drift speed. The same approach is used routinely in the MH ISR observations (Foster and Vo, 2002).

Figure 6 shows the results of a comparison between the NZ and BI plasma drift speeds in the same format as in Fig. 5. Instead of showing the scatter plot (which is extremely busy), in this presentation a 2-D occurrence distribution diagram is used. The colour of each cell in the plot represents the difference between counts detected by the NZ and BI radars according to the colour scheme in panel (a). The bin widths were $2 \mathrm{~min}$ in time and $50 \mathrm{~m} / \mathrm{s}$ in plasma drift speed. If this number is positive (red to yellow) this means that the NZ radar detected more echoes with given values of speed and during given 2-min interval. Otherwise, the BI radar detected more echoes (green to dark blue). To compare velocities for directions closest to $L$-shell where the uncertainties in the speed estimates in this method are minimal, the BI velocities in beams $7-15$ only were counted ( $L$-shell angles $\left.50^{\circ}-80^{\circ}\right)$. To compare with mostly negative NZ velocities, the BI velocities were reversed.

One can notice that an increase in the BI velocity precedes that in the NZ velocity near substorm onset in panel (a) with negative difference in counts (green cells) peaking $\sim 20 \mathrm{~min}$ after substorm and $10 \mathrm{~min}$ before the positive difference (red cells) peaked. The opposite effect is observed in panel (b), with the BI velocities (green cells) exhibiting an increase only $\sim 50 \mathrm{~min}$ after substorm onset, in sharp contrast with the NZ velocities (red and yellow cells) that reach $800-1000 \mathrm{~m} / \mathrm{s} 10-20 \mathrm{~min}$ after substorm in agreement with Fig. 5f. One can conclude that the above delays are not simply an artefact of the fitting procedure. As will be argued in Sect. 3.2, these delays are likely to be due to the finite propagation time from the substorm injection location to the position of the radars.

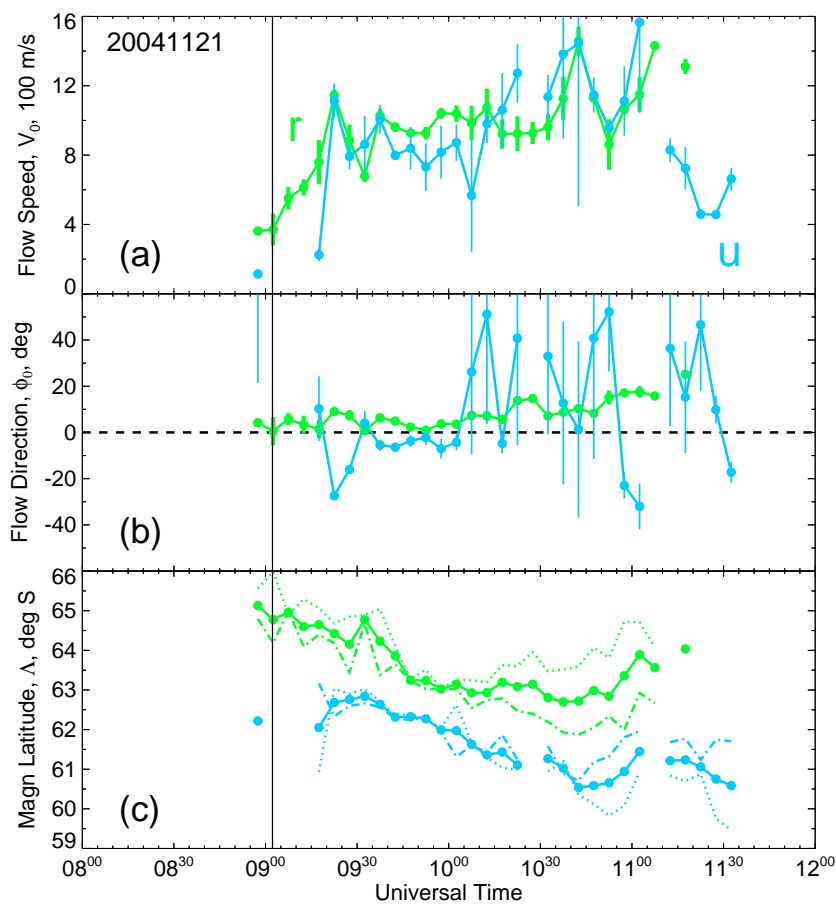

Fig. 4. Time variation of (a) the fitted velocity $V_{0}$, (b) the $L$-shell angle $\phi_{0}$, and (c) the magnetic latitude $\Lambda$ on 21 November 2004 for TIGER BI (green, "r") and NZ (blue, "u"). The fitting uncertainties are indicated by the vertical error bars. The dotted (dash-dotted) lines in panel (c) are the estimates of the flow channel location (in $\Lambda$ ) for the low- (high-) number radar beams 0-5 (10-15). The vertical line shows the substorm onset.

\section{Discussion}

3.1 On the nature of high-velocity plasma flows seen by TIGER

The high plasma flow velocities of $\sim 1500 \mathrm{~m} / \mathrm{s}$ observed by the newly-deployed zonally-looking TIGER New Zealand radar at magnetic latitudes of $\Lambda=59^{\circ}-66^{\circ} \mathrm{S}$ are a striking feature of the F-region plasma convection. They exhibit significant variability in the flow speed (and hence in the electric field strength) and latitudinal extent, Figs. 1 and 2. Comparison with the simultaneously observed high-velocity flows by the TIGER Bruny Island revealed that these flows are undoubtedly the same feature observed previously with the TIGER BI radar (Parkinson et al., 2003). The innovation of this study is that we report the first dual radar observations of the SAPS-like plasma flows. These observations provide important information on the time evolution of the subauroral electric fields as these are estimated on a quasi-continuous basis in the two closely-separated areas so that time delays and propagation associated with the flow channel formation can be investigated. This study also takes the first step towards the proper superposed epoch analysis of the substormassociated subauroral electric fields. 


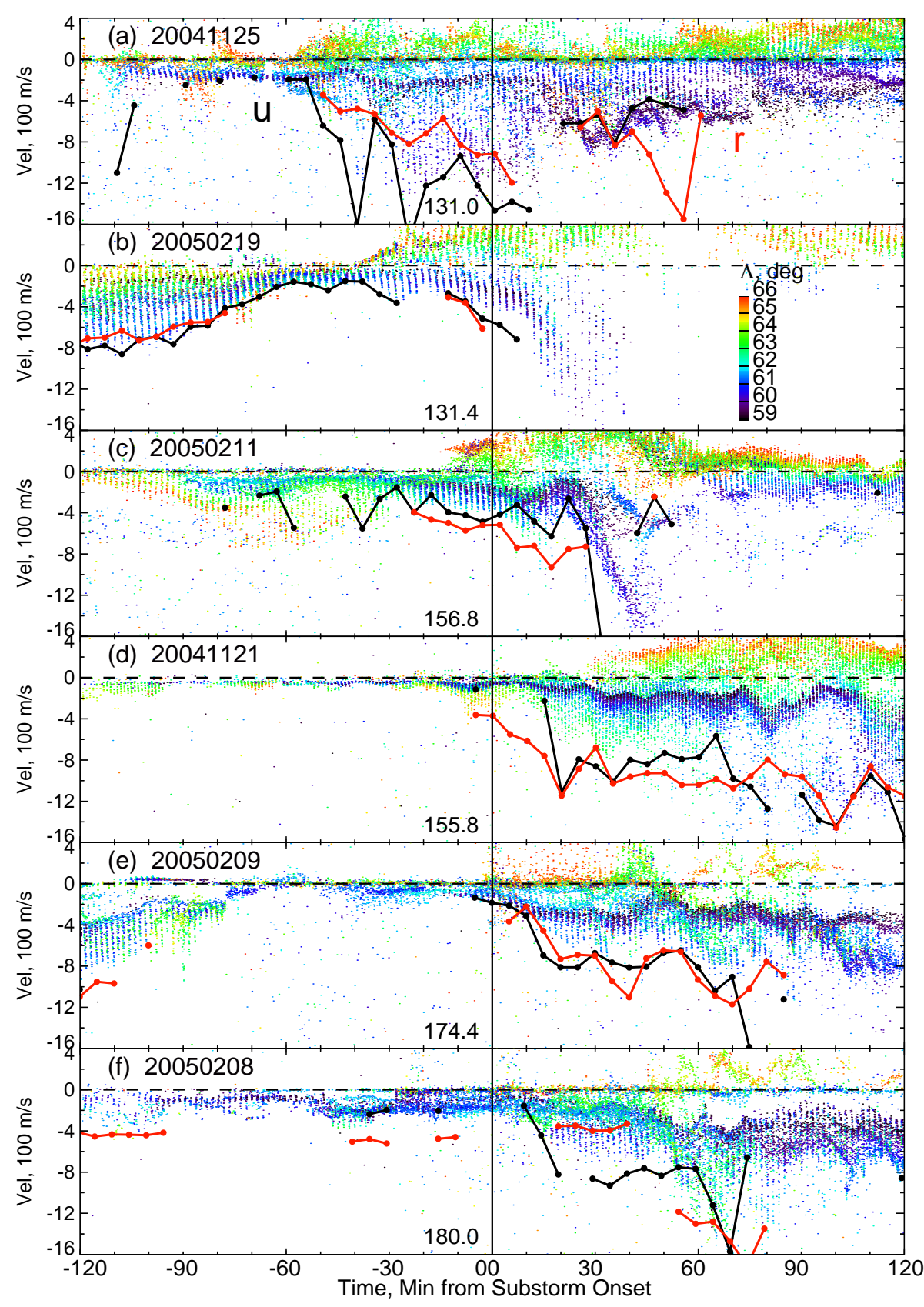

Fig. 5. Scatter plot of the TIGER NZ 1-o-s velocity versus time from substorm onset (vertical line) for all events. The points are colour-coded in magnetic latitude $\Lambda$ as shown in panel (b). Time variations of the flow speed $V_{0}$ for TIGER NZ and BI are shown by the black and red lines, respectively (the same as blue and green lines in Fig. 4a). The geographic longitude of each substorm onset location is shown to the left of the vertical line.

The first important observation is that the enhanced flows were seen equatorward of the electron precipitation region as the DMSP observations showed. Because of the limited DMSP coverage, there was no exact coincidence between the DMSP cross-track ion drift velocities and the TIGER 1-o-s irregularity velocities. One has to bear in mind also that even with the best experimental configuration used for comparison (highly homogeneous flows and closely-matched look directions) no exact match is seen (Drayton et al., 2005). However, striking similarities between the latitudinal profiles and strengths of the flow channel as seen by DMSP and TIGER demonstrate that a flow channel can become a classical PJ/SAID at later stages of its evolution (Fig. 2d) and hence should be incorporated into the definition of SAPS. A conclusion similar to the latter has been recently reached by Oksavik et al. (2006) for observations of the flow channels 
at $\Lambda=57^{\circ}-61^{\circ} \mathrm{N}$ with the Wallops Island SD radar. The magnetic latitudes in the range $61^{\circ}-64^{\circ} \mathrm{S}$, the corresponding typical $K_{p}$ values of 3-4 and generally equatorward motion are all in good agreement with the average characteristics of SAPS for this time sector reported by Foster and Vo (2002) (see their Fig. 3).

The second result of this study is that the SAPS appears to exist for at least two substorm cycles. In all events but one, clear signatures of the flow channel were observed long before the substorm onset, Fig. 5, even though for most events velocities were significantly reduced during the growth phase (Fig. 5b, e, f). For one event, when no clear signature of the flow channel was observed before the substorm onset (Fig. 5d), the DMSP ion drift observations revealed that a very weak $(\sim 200 \mathrm{~m} / \mathrm{s})$ but narrow, SAID-like signature existed just before the substorm onset. Recently Mishin and Mishin (2007) presented one example of a storm-time substorm event when a pre-existing SAPS intensified within $\sim 10$ min after the onset. Earlier Anderson et al. (1993) argued that a weak, "fossil"-like channel with marginally enhanced convection can remain dormant from previous substorm cycle and be capable of increasing rapidly in the electric field strength when a newly-generated polarization electric field is imposed on the low-conductivity region after the next substorm injection. A related feature of fossil trough in the nighttime $\mathrm{F}$ region formed by the substorm-related enhancements in the dusk-side convection and then persisting through the midnight sector was described by Evans et al. (1983). It is possible that this mechanism is at play more often than originally thought, which could explain our observations, although more modelling effort is required to answer this question definitively. Our observations also suggest that care should be exercised when introducing a threshold in the plasma drift speed/electric field as one of the criteria for PJ/SAID identification. The lower-than-threshold flows may well have existed before the substorm onset while being essentially the same phenomenon. Our observations thus support the idea that it makes more sense to talk about the time-varying electric fields within SAPS rather than about any feature that exhibits large enough convection velocity and hence has a clear onset. This is in line with a more relaxed definition of SAID proposed recently by Huang et al. (2006) according to which SAID are simply latitudinally narrow $\left(1^{\circ}-2^{\circ}\right)$ "spiky" intensifications of the westward flow irrespectively of their velocity magnitude.

\subsection{SAPS electric fields near substorm onset}

In all events but one, the plasma velocity started to increase near substorm onset. In 4 events, this increase occurred after onset, while in one event, Fig. $5 \mathrm{~b}$, the increase preceded the substorm onset by $5-10 \mathrm{~min}$. This result is opposite to that reported for auroral, reconnection-related convection near substorm onset in the midnight sector that exhibits an enhancement a few minutes before the substorm onset and

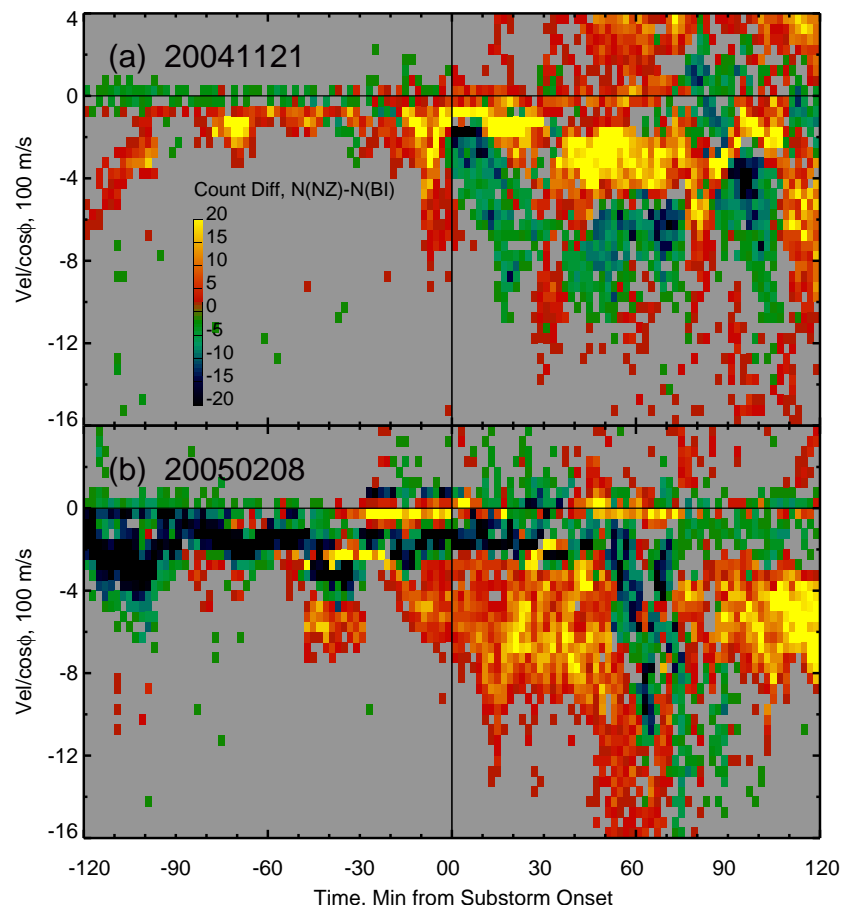

Fig. 6. 2-D colour histogram of the difference in occurrence between the TIGER NZ and BI plasma drift speeds (1-o-s velocity divided by the cosine component of the $L$-shell angle $\phi$ ) versus time from substorm onset (vertical line) for two events. Each 2-min-wide and 50-m/s-high plot cell shows the difference between the counts observed by the TIGER NZ and BI by the colour of the cell according to the colour scheme in panel (a). The BI velocities were reversed and measurements in beams $7-15$ were counted.

a marked decrease at the substorm onset (see, for example, recent paper by Bristow and Jensen, 2007, and references therein). This suggests that the plasma convection at subauroral latitudes is driven by a mechanism different from tailreconnection. Although no estimates of the 2-D plasma velocity as a function of time were reported previously, our results are consistent with the l-o-s velocity variation presented by Koustov et al. (2006) for one event observed with the King Salmon SD radar that showed a velocity increase starting at the substorm onset (see their Fig. 5a). It has been also shown that the azimuthal velocities are maximized 10-20 min after substorm in the late afternoon sector (Provan et al., 2004) and near 21:00 MLT on a statistical basis (Koustov et al., 2006).

In our observations, the only event that did not exhibit any noticeable increase near substorm onset was 25 November 2004, Fig. 5a. In fact, this scatter plot and examination of the velocity maps and velocity-angle plots analogous to Fig. 3 suggest that some decrease was seen. The substorm onset identification at 10:06 UT by IMAGE is supported by Los Alamos National Laboratory (LANL) geostationary satellite observations of energetic particles. These showed a clear dispersionless injection at this time detected by the LANL- 


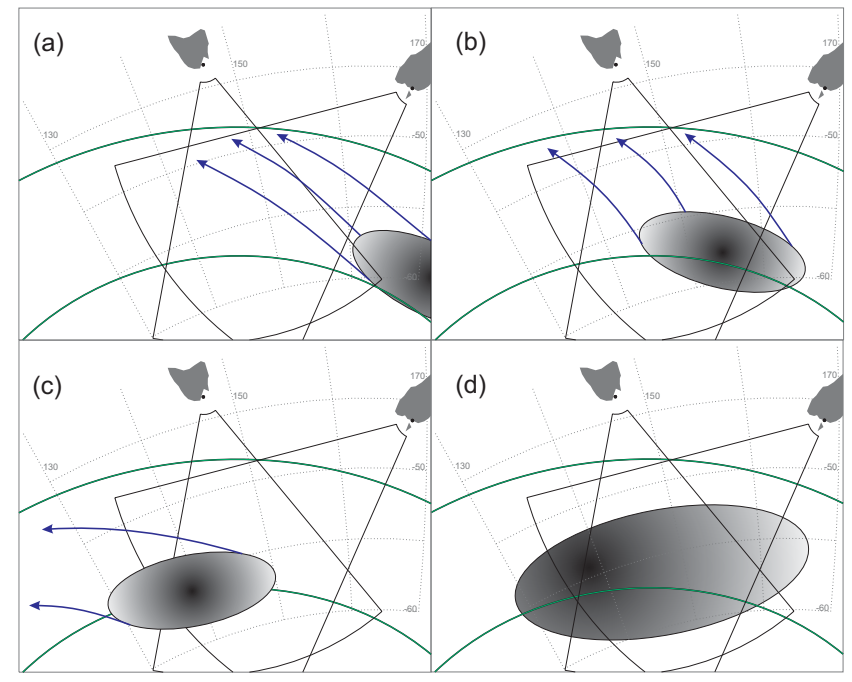

Fig. 7. Schematics of the particle injection location (gradient-filled oval) (a) eastward of, (b) within, (c) westward of, and (d) extending across the TIGER FoV. The energetic ions drift at an angle with respect to magnetic $L$ shells (green) following the blue arrows.

97A and 1994-084 satellites. Another dispersionless injection was seen by the same pair of satellites at $\sim 9: 13$ UT or $53 \mathrm{~min}$ before the substorm onset, which is in good agreement with the time when the velocity increase was observed by TIGER, Fig. 5a. AE index variation and magnetometer observations on Macquarie Island (not shown here) also support the idea that another substorm has occurred 53 min before the one at 10:06 UT. Thus the absence of a clear velocity increase near 10:06 UT is unlikely to be due to an error in the substorm onset timing and is possibly due to this substorm not being isolated with the velocity variation being affected by the previous substorm cycle.

Analysis of events with a velocity increase near substorm onset, Fig. 5b-f, showed that the response time of the electric field within the flow channel to the substorm onset was variable from -5 (Fig. 5b) to +40 min (Fig. $5 f$ for BI radar). It was noted that this response time appeared to depend on the location of the substorm onset with longer delays observed for substorm onsets eastward of the TIGER FoV. As geographic longitude of the substorm onset increased from Fig. 5b to Fig. 5f, the delays were generally larger. Thus almost instantaneous (within $5 \mathrm{~min}$ ) responses were observed by the BI radar in Fig. 5b-d, whereas in Fig. 5e and $\mathrm{f}$ the response times were considerably longer. This was generally the case for the NZ radar as well although the largest delay was observed in Fig. 5d. It was also noted that the BI velocity increase preceded that in the NZ velocity when substorm onsets occurred within or westward of the TIGER FoV, Fig. 5b-d. The fact that the delay in the NZ velocity in Fig. 5d was the largest or at least comparable to that in Fig. 5f, while the substorm onset location was much closer in zonal direction, suggests that factor(s) other than simply dis- tance in the zonal direction may be important. From Fig. 2b, no high-velocity echoes were seen by the NZ radar eastward and equatorward of the substorm onset location with simultaneous high-velocity echoes observed by the BI radar westward and equatorward of that. This suggests that the propagation velocity of the region with high electric fields was predominantly westward in this case. Westward propagation velocity is also suggested by Fig. 5e and f.

Our observations support the notion that high-velocity plasma flows are driven by the polarization electric field due to charge separation during substorm development as discussed below. After the substorm injection the energetic protons penetrate deeper into the inner magnetosphere than the electrons under the combined action of the convection electric field and the curvature-gradient drift (Southwood and Wolf, 1978). The protons from the initial injection region drift westward and inward/equatorward near magnetic midnight (e.g. Galperin, 2002). Consequently the region with enhanced electric field should expand westward and equatorward in the midnight sector of interest.

Figure 7 illustrates this situation showing the injection region, the proton trajectories and the TIGER FoV for four cases. In case (a), the initial injection region shown by the gradient-filled oval is eastward of the TIGER FoV. The injected protons drift westward and equatorward, enter the TIGER FoV and the polarization field is established with some delay. The delays between the velocity increases measured by the two radars will vary depending on the magnetic latitude and shape of the injection region but generally TIGER NZ should detect an increase sooner. This case refers to Fig. $5 e$ and $f$. In case (b), the protons from the injection region enter the TIGER BI FoV at subauroral latitudes first with the protons from the equatorward and eastward edge (upper blue arrow) following, which results in the BI velocity increase preceding that of the NZ velocity, Fig. $5 \mathrm{c}$ and d. In case (c), the injection is westward of the TIGER FoV and no polarization field is observed there, which possibly refers to Fig. 5a. Finally, when the centre of the substorm onset is also westward but the injection region is sufficiently large to extend across both TIGER BI and NZ FoVs, panel (d), the polarization field is established almost simultaneously in these areas and this is a possible explanation for the data presented in Fig. 5b.

The time evolution of the electric fields after the initial increase "phase" was similar as seen by both TIGER radars in most events. After the initial increase, the velocity was either reaching the maximum monotonically as in Fig. $5 \mathrm{~b}$ and $\mathrm{c}$ or more gradually with some variations as in Fig. $5 \mathrm{~d}-\mathrm{f}$. The maximum velocity was observed a few tens of min after the substorm onset, from $20 \mathrm{~min}$ in Fig. 5 b to over $90 \mathrm{~min}$ in Fig. 5d. This result is in agreement with that reported by Koustov et al. (2006).

Our observations suggest that the time variation of the electric field within the SAPS is a two-stage process. During the first phase, the velocity is mostly controlled by the 


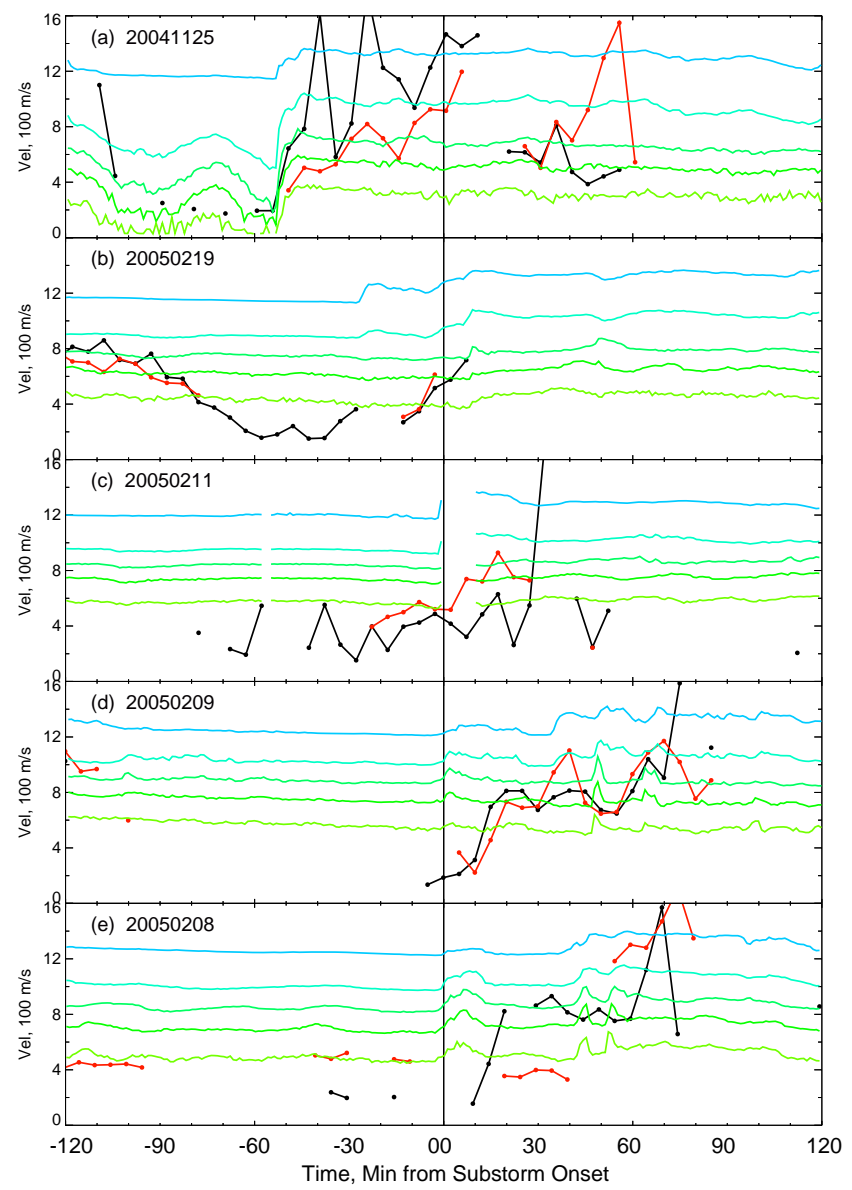

Fig. 8. Time variation of the TIGER BI (red) and NZ (black) velocities (the same as in Fig. 5 but reversed) and the LANL 1994084 satellite fluxes in arbitrary logarithmic scale. The proton fluxes shown are (from top to bottom): 50-75, 75-113, 113-170, 170250 , and $250-400 \mathrm{keV}$.

polarization electric field being established in the subauroral ionosphere. In the second phase, the electric field variation is likely to be affected by the ionosphere-magnetosphere positive feedback processes during which the strong electric field decreases the conductance, with the decreasing conductance reinforcing the electric field (see, for example, the recent review by Wolf et al., 2007, and references therein). Consequently, how the electric field will vary with time at a particular location in the second phase may depend on the ionospheric conditions at this location. Similar processes are believed to be responsible for time variation of the SAPS electric fields during substantial geomagnetic storms with the "main" SAPS electric field produced by the radial separation between ions and electrons associated with ring current enhancement and with rapidly-changing SAID-like perturbations produced by the ionospheric feedback instability (e.g. Foster et al., 2004; Oksavik et al., 2006).

A possible complicating factor is additional injections of the energetic ions during substorm intensifications following the main substorm injection which can reinforce the polarization electric field. To check whether any such additional injections occurred in our events, we analyzed the proton fluxes measured by the Synchronous Orbit Particle Analyzer (SOPA) onboard of the geostationary satellite LANL 1994084, a closest satellite in MLT to our observational sector. Figure 8 shows the TIGER fitted velocities (reversed as compared to Fig. 5) and the proton fluxes in an arbitrary logarithmic scale for the 5 events for which the LANL 1994-084 proton fluxes were available. The first feature to note is a dispersionless injection of protons at $-53 \mathrm{~min}$, Fig. $8 \mathrm{a}$, observed almost simultaneously with the increase in TIGER NZ velocity. This observation once again confirms our conclusion that the velocity increase before substorm onset at 10:06 UT was due to another substorm that occurred $53 \mathrm{~min}$ earlier. The second observation is that the vertical line corresponds to a dispersionless proton injection for every event for which a velocity increase was seen as expected. One should note that there is a dispersionless injection in the SOPA electron data for the first event as well (not shown) with no proton counterpart, Fig. 8a, which is a possible reason why no velocity increase was observed for this event. An interesting feature is observed in Fig. 8b where proton fluxes start to increase $\sim 5 \mathrm{~min}$ before the substorm onset. This was also the event when the velocity increase occurred 5-10 min before the substorm, which provides an important clue to why this may have happened. Sergeev et al. (1998) have reported observations of the two-layer injection front detected with the CRRES satellite at $5 R_{E}$ with a dispersionless proton front preceding that of the electrons. The time delay was $4 \mathrm{~min}$ which is in good agreement with our observations. This provides additional support to the direct association between the plasma velocity increase and proton injections near substorm onset. Fig. 8d and e also show that the velocity increases detected by both TIGER radars $\sim 55$ and 60 min after the substorm onset, in events shown in panels (d) and (e), respectively, were preceded by the dispersionless increases in the proton flux $\sim 45$ and 42 min after the substorm onset, in panels (d) and (e), respectively, which suggests that the association between the plasma velocity increase and proton injections may exist for subsequent intensification/proton injections as well.

Similar to the SAPS electric fields, the meridional motions of the SAPS peak also exhibited some variability. Analysis of the diagrams analogous to Fig. 4c for all 6 events (not presented here) shows that in two events (21 and 25 November 2004), the SAPS steadily migrated equatorward, while in other two (8 and 9 February 2005) it was mostly stationary in magnetic latitude. The SAPS equatorward migration is not unexpected and can be explained by the energetic protons penetrating to lower L shells. One must bear in mind though that some (or all) of it can be an apparent effect due to the SAPS being at more equatorward locations at later MLTs (Foster and Vo, 2002). As the radars move towards magnetic midnight they sample the SAPS at more equatorward 
locations. The absence of any substantial movement in some events is difficult to explain through either of these effects but may be, again, suggestive of the two-stage nature of the SAPS development with the ionosphere-magnetosphere coupling processes possibly playing a substantial role in the second stage.

\section{Summary and Conclusions}

The first quasi-continuous observations of the localized highvelocity F-region plasma flows at $\Lambda=59^{\circ}-66^{\circ} \mathrm{S}$ by the dual TIGER HF radars near substorm onset were presented. Six events were selected during which TIGER detected both a large number of high-velocity echoes and a substantial number of echoes near the substorm onset. A comparison with the simultaneously observed DMSP signatures showed that the flow channels extend equatorward of the electron precipitation region and that they can become classical PJ/SAID at later stages of their evolution. Hence it was argued that AWFC should be incorporated into a definition of SAPS.

A semi-automatic method of the plasma velocity derivation was developed using iterative fitting to the maximum velocities detected within SAPS. Analysis of the time variation of the plasma velocity around substorm onset showed that the SAPS appeared not to have a clear onset, rather they existed in a dormant state before the substorm onset. The plasma velocity typically exhibited an increase near substorm onset with variable response times, from -5 to $+40 \mathrm{~min}$. These results suggested that the SAPS channel exists independently of the substorm-related processes which simply reinforce the plasma convection after the substorm onset. The result showing plasma velocity increase after substorm onset was opposite to that reported for auroral convection in the midnight sector for which a sharp decrease at the substorm onset is seen. This suggested that the plasma convection at auroral and subauroral latitudes is driven by different mechanisms.

The shape of velocity variation with time and the response time to the substorm onset varied from event to event, with some events showing a substantial monotonic increase within 20-40 min from the substorm onset and with others showing a more gradual increase with some variation. The response times exhibited some dependence on the substorm onset location with longer delays observed for substorm onsets that were detected eastward of TIGER. The analysis of the response times detected by the two closelyseparated TIGER radars suggested that the region with enhanced velocities expands westward and equatorward following the energetic ions that drift from the substorm injection region. Examination of the LANL proton fluxes measured at geostationary orbit showed that the velocity increase was associated with the dispersionless ion injection including the case when the velocity increase started before the substorm onset (negative response time). The time variation of the electric fields within the SAPS suggests that it is a two-stage process. The initial velocity increase is associated with the establishment of the polarization electric field near substorm onset with delays dependent on the location of the ion injection region. The time evolution following the initial increase may depend on both the ionospheric conditions through the ionosphere-magnetosphere coupling processes and additional supply of energetic ions during subsequent intensifications.

Acknowledgements. This research was supported by the Australian Research Council Discovery grant to R.A.M. (project DP0770366). TIGER is supported by the ARC Discovery grant DP0664424, the Australian Antarctic Science grant program, and a consortium of institutions: La Trobe University, University of Newcastle, Monash University, Australian Government Antarctic Division, ISR Division DSTO and IPS Radio and Space Services. Additional funding towards construction of the TIGER radars was received from the ARC, US Air Force Office of Scientific Research, British Antarctic Survey and RLM Systems Pty Ltd, Australia. The authors gratefully acknowledge the Center for Space Sciences at the University of Texas at Dallas and the US Air Force for providing the DMSP thermal plasma data. The DMSP particle detectors were designed by D. Hardy of AFRL, and data obtained from JHU/APL. The authors thank D. Hardy, F. Rich, and P. Newell for its use. D. DeLapp and G. Reeves of Los Alamos National Laboratory are thanked for providing the SOPA LANL particle flux data. R. A. Makarevich thanks A. V. Koustov and J.-P. St.-Maurice for fruitful discussions.

Topical Editor Mike Pinnock thanks K. Oksavik and another anonymous referee for their help in evaluating this paper.

\section{References}

Anderson, P. C., Hanson, W. B., Heelis, R. A., Craven, J. D., Baker, D. N., and Frank, L. A.: A proposed production model of rapid subauroral ion drifts and their relationship to substorm evolution, J. Geophys. Res., 98, 6069-6078, 1993.

Bristow, W. A. and Jensen, P.: A superposed epoch study of SuperDARN convection observations during substorms, J. Geophys. Res., 112, A06232, doi:10.1029/2006JA012049, 2007.

Chisham, G., Lester, M., Milan, S. E., Freeman, M. P., Bristow, W. A., Grocott, A., McWilliams, K. A., Ruohoniemi, J. M., Yeoman, T., Dyson, P. L., Greenwald, R. A., Kikuchi, T., Pinnock, M., Rash, J. P. S., Sato, N., Sofko, G. J., Villain, J.P., and Walker, A. D. M.: A decade of the Super Dual Auroral Radar Network (SuperDARN): scientific achievements, new techniques and future directions, Surv. Geophys., 28, 33-109, doi:10.1007/s10712-007-9017-8, 2007.

Drayton, R. A., Koustov, A. V., Hairston, M. R., and Villain, J.P.: Comparison of DMSP cross-track ion-drifts and SuperDARN line-of-sight velocities, Ann. Geophys., 23, 2479-2486, 2005, http://www.ann-geophys.net/23/2479/2005/.

Evans, J. V., Holt, J. M., Oliver, W. L., and Wand, R. H.: The fossil theory of nighttime high latitude $\mathrm{F}$ region troughs, J. Geophys. Res., 88, 7769-7782, 1983.

Foster, J. C. and Burke, W. J.: SAPS: A new categorization for subauroral electric fields, EOS, 83, 393-394, 2002.

Foster, J. C. and Vo, H. B.: Average characteristics and activity dependence of the subauroral polarization stream, J. Geophys. Res., 17, 1475, doi:10.1029/2002JA009409, 2002. 
Foster, J. C., Erickson, P. J., Lind, F. D., and Rideout, W.: Millstone Hill coherent-scatter radar observations of electric field variability in the Sub-Auroral Polarization Stream, Geophys. Res. Lett., 31, L21803, doi:10.1029/2004GL021271, 2004.

Freeman, M. P., Southwood, D. J., Lester, M., Yeoman, T. K., and Reeves, G. D.: Substorm-associated radar auroral surges, J. Geophys. Res., 97, 12 173-12 185, 1992.

Frey, H. U. and Mende, S. B.: Substorm onset as observed by IMAGE-FUV, Proceedings of the 8th International Conference on Substorms, 71-75, 2006.

Galperin, Y. I.: Polarization jet: Characteristics and a model, Ann. Geophys., 20, 391-404, 2002, http://www.ann-geophys.net/20/391/2002/.

Galperin, Y. I., Ponomarev, V. N., and Zosimova, A. G.: Direct measurements of ion drift velocity in the topside ionosphere during a magnetic storm, Cosmicheskie Issled., 11, 273-283, 1973.

Greenwald, R. A., Baker, K. B., Dudeney, J. R., Pinnock, M., Jones, T. B., Thomas, E. C., Villain, J.-P., Cerisier, J.-C., Senior, C., Hanuise, C., Hunsuker, R. D., Sofko, G., Koehler, J., Nielsen, E., Pellinen, R., Walker, A. D. M., Sato, N., and Yamagishi, H.: DARN/SuperDARN: A global view of the dynamics of highlatitude convection, Space Sci. Rev., 71, 763-796, 1995.

Huang, C., Sazykin, S., Spiro, R., Goldstein, J., Crowley, G., and Ruohoniemi, J. M.: Storm-time penetration electric fields and their effects, EOS, 87, 131-132, 2006.

Karlsson, T., Marklund, G. T., and Blomberg, L. G.: Subauroral electric fields observed by the Freja satellite: A statistical study, J. Geophys. Res., 103, 4327-4341, 1998.

Koustov, A. V., Drayton, R. A., Makarevich, R. A., McWilliams, K. A., St-Maurice, J.-P., Kikuchi, T., and Frey, H. U.: Observations of high-velocity SAPS-like flows with the King Salmon SuperDARN radar, Ann. Geophys., 24, 1591-1608, 2006, http://www.ann-geophys.net/24/1591/2006/.

Makarevitch, R. A., Honary, F., and Koustov, A. V.: Simultaneous $\mathrm{HF}$ measurements of E- and F-region Doppler velocity at large flow angles, Ann. Geophys., 22, 1177-1188, 2004, http://www.ann-geophys.net/22/1177/2004/.
Mishin, E. V. and Mishin, V. M.: Prompt response of SAPS to stormtime substorm, J. Atmos. Sol. Terr. Phys., 69, 1233-1240, 2007.

Oksavik, K., Greenwald, R. A., Ruohoniemi, J. M., Hairston, M. R., Paxton, L. J., Baker, J. B. H., Gjerloev, J. W., and Barnes, R. J.: First observations of the temporal/spatial variation of the sub-auroral polarization stream from the SuperDARN Wallops HF radar, Geophys. Res. Lett., 33, L12104, doi: 10.1029/2006GL026256, 2006.

Parkinson, M. L., Pinnock, M., Ye, H., Hairston, M. R., Devlin, J. C., Dyson, P. L., Morris, R. J., and Ponomarenko, P.: On the lifetime and extent of an auroral westward flow channel (AWFC) observed during a magnetospheric substorm, Ann. Geophys., 21, 893-913, 2003, http://www.ann-geophys.net/21/893/2003/.

Provan, G., Lester, M., Mende, S. B., and Milan, S. E.: Statistical study of high-latitude plasma flow during magnetospheric substorms, Ann. Geophys., 22, 3607-3624, 2004, http://www.ann-geophys.net/22/3607/2004/.

Ruohoniemi, J. M., Greenwald, R. A., Baker, K. B., Villain, J.-P., and Hanuise, C.: Mapping high-latitude plasma convection with coherent HF radars, J. Geophys. Res., 94, 13 463-13 477, 1989.

Sergeev, V. A., Shukhtina, M. A., Rasinkangas, R., Korth, A., Reeves, G. D., Singer, H. J., Thomsen, M. F., and Vagina, L. I.: Event study of deep energetic particle injections during substorm, J. Geophys. Res., 103, 9217-9234, 1998.

Southwood, D. J. and Wolf, R. A.: An assessment of the role of precipitation in magnetospheric convection, J. Geophys. Res., 83, 5227-5232, 1978.

Spiro, R. W., Heelis, R. A., and Hanson, W. B.: Rapid subauroral ion drifts observed by Atmospheric Explorer C, Geophys. Res. Lett., 6, 657-660, 1979.

Unwin, R. S. and Cummack, C. H.: Drift-spikes; The ionospheric signature of large poleward directed electric fields at subauroral latitudes, Mem. Nat. Inst. Polar Res., 16, 72-83, 1980.

Wolf, R. A., Spiro, R. W., Sazykin, S., and Toffoletto, R.: How the Earth's inner magnetosphere works: An evolving picture, J. Atmos. Sol. Terr. Phys., 69, 288-302, 2007. 